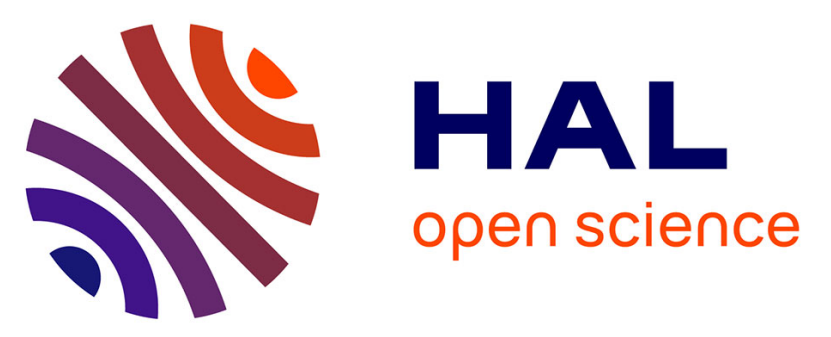

\title{
Total metabolic tumor volume and spleen metabolism on baseline [18F]-FDG PET/CT as independent prognostic biomarkers of recurrence in resected breast cancer
}

Romain-David Seban, Roman Rouzier, Aurelien Latouche, Nicolas Deleval, Jean-Marc Guinebretiere, Irene Buvat, Francois-Clement Bidard, Laurence

Champion

\section{To cite this version:}

Romain-David Seban, Roman Rouzier, Aurelien Latouche, Nicolas Deleval, Jean-Marc Guinebretiere, et al.. Total metabolic tumor volume and spleen metabolism on baseline [18F]-FDG PET/CT as independent prognostic biomarkers of recurrence in resected breast cancer. European Journal of Nuclear Medicine and Molecular Imaging, 2021, 48 (11), pp.3560-3570. 10.1007/s00259-021-05322-2 . hal-03441739

\author{
HAL Id: hal-03441739 \\ https://hal.science/hal-03441739
}

Submitted on 1 Dec 2021

HAL is a multi-disciplinary open access archive for the deposit and dissemination of scientific research documents, whether they are published or not. The documents may come from teaching and research institutions in France or abroad, or from public or private research centers.
L'archive ouverte pluridisciplinaire $\mathbf{H A L}$, est destinée au dépôt et à la diffusion de documents scientifiques de niveau recherche, publiés ou non, émanant des établissements d'enseignement et de recherche français ou étrangers, des laboratoires publics ou privés. 
Total metabolic tumor volume and spleen metabolism on baseline [18F]-FDG PET/CT as independent prognostic biomarkers of recurrence in resected breast cancer

Romain-David Seban ${ }^{1,2}$, Roman Rouzier ${ }^{3}$, Aurélien Latouche ${ }^{4,5}$, Nicolas Deleval ${ }^{1}$, Jean-Marc Guinebretiere ${ }^{6}$ , Irène Buvat ${ }^{2}$, Francois-Clément Bidard ${ }^{7,8}$, Laurence Champion ${ }^{1,2}$

1. Department of Nuclear Medicine, Institut Curie, 92210 Saint-Cloud, France

2. Laboratoire d'Imagerie Translationnelle en Oncologie, Inserm U1288, PSL Research University, Institut Curie, 91400 Orsay, France

3. Department of Surgery, Institut Curie, PSL Research University, 75005 Paris \&, 92210 Saint-Cloud, France

4. Bioinformatics and Computational Systems Biology of Cancer, PSL Research University, Mines Paris Tech, INSERM U900, 75005 Paris, France

5. Conservatoire national des arts et métiers, Paris, France

6. Department of Pathology, Institut Curie, 92210 Saint-Cloud, France

7. Department of Medical Oncology, Institut Curie, PSL Research University, 75005 Paris \&, 92210 SaintCloud, France

8. Circulating Tumor Biomarkers Laboratory, SiRIC, Institut Curie, PSL Research University, Paris, France 


\begin{abstract}
:
Purpose: We evaluated whether biomarkers on baseline [18F]-FDG PET/CT are associated with recurrence after surgery in patients with invasive breast cancer of no special type (NST).
\end{abstract}

Methods: In this retrospective single-center study, we included consecutive patients with non-metastatic breast cancer of NST who underwent [18F]-FDG PET/CT before treatment, including surgery, between 2011 and 2016. Clinicopathological data were collected. Tumor SUVmax, total metabolic tumor volume (TMTV), and spleen- and bone marrow-to-liver SUVmax ratios (SLR, BLR) were measured from the PET images. Cut-off values were determined using predictiveness curves to predict 5-year recurrence-free survival (5y-RFS). A multivariable prediction model was developed using Cox regression. The association with stromal tumorinfiltrating lymphocytes (TILs) levels (low if $<50 \%$ ) was studied by logistic regression.

Results: Three hundred and three women were eligible, including 93 (31\%) with triple-negative breast carcinoma. After a median follow-up of 6.2 years, 56 and 35 patients experienced recurrence and death, respectively. The 5y-RFS rate was $86 \%$. In multivariable analyses, high TMTV $(>20 \mathrm{~cm} 3)$ and high SLR $(>0.76)$ were associated with shorter 5y-RFS (HR 2.4, 95\%CI 1.3-4.5, and HR 1.9, 95\%CI 1.0-3.6). In logistic regression, high SLR was the only independent factor associated with low stromal TILs (OR 2.8, 95\%CI 1.45.7).

Conclusion: High total metabolic tumor volume and high spleen glucose metabolism on baseline [18F]-FDG $\mathrm{PET} / \mathrm{CT}$ were associated with poor 5y-RFS after surgical resection in patients with breast cancer of NST. Spleen metabolism was inversely correlated with stromal TILs and might be a surrogate for an immunosuppressive tumor microenvironment.

Keywords: Invasive breast cancer of no special type - [18F]-FDG PET/CT - Prognosis - Total metabolic tumor volume - Spleen glucose metabolism - Stromal tumor-infiltrating lymphocytes 


\section{Introduction}

Identification of more accurate predictors of recurrence in patients with non-metastatic breast cancer might facilitate the selection of therapy. Treatment decisions are currently based on clinicopathological characteristics as well as tools that provide additional information in the decision-making process, such as genomic tests or molecular signatures [1-3]. However, these tests are not readily available in all institutions and discordances in the estimates provided by these tests have sometimes been observed [4]. In addition, these signatures do not take into account the individual imaging characteristics of breast cancer patients.

Fluorine-18-fluorodeoxyglucose positron emission tomography/computed tomography ([18F]-FDG $\mathrm{PET} / \mathrm{CT}$ ) is currently recommended by the National Comprehensive Cancer Network (NCCN) guidelines for disease staging $[5,6]$ and might also provide significant prognostic information $[7,8]$. Tumor glucose metabolism assessed by the maximum standardized uptake value (SUVmax) is used as a surrogate of tumor aggressiveness and tumor cell proliferation $[9,10]$. Several studies have demonstrated the prognostic value of SUVmax of the primary breast tumor and/or regional lymph nodes measured on [18F]-FDG PET/CT in nonmetastatic breast cancer [11-13]. There is also strong evidence that metabolic tumor volume, as measured by total metabolic tumor volume (TMTV) or total lesion glycolysis (TLG), is a strong predictor of recurrence [14, 15] and pathological complete response in the subset of patients treated by neoadjuvant chemotherapy [16].

An original approach to the prognosis of patient outcomes does not focus exclusively on tumor imaging characteristics but includes imaging assessment of lymphoid tissues [17-21]. In the era of precision medicine, such an approach might provide novel prognostic biomarkers to guide breast cancer patient management [2224]. Two main types of imaging biomarkers can be extracted from baseline [18F]-FDG PET/CT for this purpose: tumor metabolism (tumor uptake and tumor volume) and nontumoral lymphoid tissue metabolism (spleen and bone marrow). In addition to tumor tissue, nontumoral lymphoid tissues are reliable candidates, as bone marrow (BM) and spleen FDG uptake measurements are considered to be surrogates of cancer-related inflammation, which may have prognostic significance in a broad spectrum of tumor types [17, 18, 20, 25-27].

The current hypothesis is that tumor growth could play a pivotal role in reprogramming the host immune system by regulating hematopoiesis, which might be associated with the expansion of BM and/or spleen-derived immunosuppressive cells, such as tumor associated macrophages (TAMs) [28], regulatory $\mathrm{T}$ cells (Tregs) [29], and Myeloid-Derived Suppressor Cells (MDSCs) [30]. In the specific population of patients with invasive breast cancer of no special type (NST), only two recent studies, evaluating spleen [23] or BM [24] metabolism as prognostic factors, could possibly improve stratification risk and guide treatment strategies. However, external validation on large cohorts is an essential step before implementing these prognostic biomarkers in clinical practice. Similarly, a better understanding of the pathophysiological process is also needed to elucidate the relationship between the tumor and lymphoid tissues [31]. 
In this study, we specifically addressed this issue by determining the capacity of biomarkers measured on pretreatment [18F]-FDG PET/CT and reflecting tumor and nontumoral lymphoid tissues metabolism, to predict recurrence in addition to standard clinicopathological variables in patients with primary non-metastatic breast cancer of NST. Secondly, we assessed the association between these imaging biomarkers and histopathological findings in order to clarify the pathophysiological mechanisms involved in the cross-talk between the tumor and its microenvironment and lymphoid tissues.

\section{Methods}

\section{Patients}

We conducted a retrospective review of 355 consecutive patients with biopsy-proven invasive breast cancer of NST who underwent [18F]-FDG PET/CT imaging for initial staging at Institut Curie Hospital, SaintCloud, France (the flow chart is provided in Fig. 1). Thirty-five patients were excluded on the basis of the following criteria (some patients met multiple exclusion criteria): (i) patients with metastatic disease $(\mathrm{n}=13)$; (ii) patients with $<24$ months of follow-up without any event $(n=9)$; (iii) patients with no measurable disease or no FDG-avid tumor $(\mathrm{n}=8)$; (iv) patients with other primary malignancies $(\mathrm{n}=7)$; (v) patients with treatment or disease that could potentially influence metabolic activities in the spleen or bone marrow such as corticosteroids, lithium, G or GM-CSF received over the last 2 months, history of chronic inflammatory, autoimmune, or hematologic disease (hematologic malignancy or hemolytic anemia) or acute or chronic infection (n $=5)$; and (vi) patients who expressed their opposition to participate in medical research $(\mathrm{n}=10)$. This retrospective data collection complied with our Institutional Review Board (IRB DATA200300), with a waiver of informed consent ("rule of non-opposition") and the study was conducted in compliance with the Declaration of Helsinki.

\section{Treatment and post-treatment surveillance}

Two main treatment sequences were defined according to the administration of neoadjuvant therapy or upfront surgery: in the first group, treatment consisted of neoadjuvant therapy (chemotherapy or endocrine therapy) together with anti- HER2 therapy for HER2+ cancers, followed by surgery (breast-conserving surgery or mastectomy, with axillary lymph node dissection or sentinel lymph node technique), radiotherapy, and endocrine therapy for HR+ cancers. In the second group, treatment consisted of upfront surgery (breast conserving surgery or mastectomy, with axillary lymph node dissection or sentinel lymph node technique), followed by adjuvant chemotherapy when indicated (+ anti-HER2 therapy for HER2+ cancers), radiotherapy, and endocrine therapy for HR+ cancers. 
After treatment, patients were regularly evaluated by clinical examinations, annual mammography, and follow-up tumor marker tests. Follow-up examinations were performed every 3 months for the first 2 years, then every 6 months for the next 3 years, and annually thereafter. When recurrence was suspected, CT, MRI, or PET imaging was performed and biopsy of the lesion was recommended.

\section{Clinicopathological data}

Clinical data consisted of the patient's age and menopausal status. $\mathrm{T}$ and $\mathrm{N}$ stages were determined according to the 8th edition of the American Joint Committee on Cancer (AJCC) Staging Manual [32, 33]. Pathological characteristics of the primary breast cancer were obtained from biopsy samples, including hormone receptor status (estrogen-ER and progesterone-PR), HER2 status, Ki-67 index, mitotic index, histologic grade, and presence of vascular invasion, necrosis, or stromal tumor-infiltrating lymphocytes (TILs). Stromal TILs were assessed on hematoxylin and eosin stained sections and sections with $\geq 50 \%$ stromal TIL infiltrate were documented as breast cancer with high TILs [34], as proposed in the literature. Tumor markers (cancer antigen-CA-15-3 and carcinoembryonic antigen-CEA) were measured before treatment initiation. The whole cohort was classified according to the four main molecular subtypes (St. Gallen consensus): Luminal A (ER+/PR+/HER2-/low Ki-67); Luminal B (ER+/PR+/HER2-/+/high Ki-67); HER2-overexpression (ER-/PR/HER2+), and triple-negative breast cancers/ TNBCs (ER-/PR-/HER2-) [35].

\section{[18F]-FDG PET/CT scans}

[18F]-FDG PET/CT scans were performed as part of staging in accordance with the applicable EANM procedure guidelines [36]. Patients fasted for at least $6 \mathrm{~h}$ prior to scanning to ensure a blood glucose level $<$ $10 \mathrm{mmol} / \mathrm{L}$. Scanning was performed from the skull base to the proximal femur using a General Electric Discovery-690 (GE Healthcare, Waukesha, WI). PET images were reconstructed with a fully 3D time-of flight iterative reconstruction method (VPFX) (OSEM algorithm, 24 subsets, 2 iterations). Images were converted to SUV units by normalization using the patient's body weight.

\section{Measurement of imaging biomarkers}

A pair of board-certified nuclear medicine physicians (RDS, ND) analyzed [18F]-FDG PET/CT scans. Lesions were then segmented using the PET tumor segmentation tool in Philips IntelliSpace Portal 9.0. Readers were blinded and had no knowledge of the patient's history or pathological background, imaging features, or clinical outcome.

Tumor tissues. The primary tumor and when applicable, regional lymph nodes were delineated on the PET images as recommended by the EANM for solid tumors [36], using a SUVmax threshold of 41\%, in a 
manually drawn volume, assisted by CT data for the anatomical location to extract tumor PET parameters. A patient's SUVmax was defined as the highest SUVmax among all lesions detected in the patient, including primary and metastatic lymph nodes when applicable. TMTV was defined as the sum of the metabolic tumor volume of all lesions (primary tumor and metastatic lymph node when applicable).

Nontumoral lymphoid tissues. Liver, spleen, and BM SUVmax values were computed with the same methodology as that used in several published studies [18-20,24] by drawing a spherical $3 \mathrm{~cm}-\mathrm{VOI}$ in the liver, a spherical $2 \mathrm{~cm}$-VOI in the spleen, and four spherical $1.5 \mathrm{~cm}$-VOI placed in the center of L1 to L4 (lumbar) vertebral bodies for BM. BM SUVmax was defined as the average of the maximum standardized uptake values of all vertebral bodies. Spleen and BM uptakes were normalized by computing the spleen/liver ratio (SLR) and BM/liver ratio (BLR) respectively: spleen SUVmax was divided by liver SUVmax (SLR) and BM SUVmax was divided by the liver SUVmax (BLR).

\section{Outcomes}

The primary endpoint was recurrence-free survival (RFS), defined as the time from primary treatment (NAC or surgery) to disease relapse or death from any cause or to the date of censoring at the last time the patient was known to be alive. Secondary endpoint analyses were related to loco-regional and systemic control. Loco-regional recurrence was defined as relapse in residual breast tissue or chest wall in the case of nonconserving breast surgery, or in the ipsilateral lymph drainage area or axillary/infraclavicular/supraclavicular area. Distant recurrence was defined as all other forms of relapse. We considered the following 5-year outcome measures: recurrence-free survival (5y-RFS), loco-regional recurrence free survival (5y-LRFS), and distant recurrence-free survival (5y-DRFS). The duration of follow-up was calculated from the date of the initial [18F]-FDG PET/CT to the date of the last clinical consultation.

\section{Statistical analysis}

All patients were followed for at least 24 months or until death. Spearman's rank correlation coefficients (rho) were estimated to assess the relationships between PET parameters. Values of PET biomarkers between patients with low or high stromal TILs were compared using the Mann- Whitney-Wilcoxon test. Factors associated with stromal TILs were tested by logistic regression. Median follow-up was estimated using the reverse Kaplan-Meier method [37]. We used predictiveness curves in order to determine relevant cut-off values (considering a 5-year horizon) and to dichotomize biomarkers [38, 39]. Survival curves were estimated by the Kaplan-Meier method (log-rank test). The prognostic impact of all factors measured at diagnosis was studied with Cox proportional hazards models for survival with over time and linear dependency of the log-hazard function on covariates. The prognostic model for 5y-RFS was build using backward variable selection. The 
likelihood ratio test (LRT) for added prognostic value of TMTV and SLR was obtained by comparing log likelihoods of multivariable prognostic models without TMTV or SLR and with TMTV plus SLR (chi-square test: $\chi 2$ ). The cumulative incidence of loco-regional recurrence was estimated considering death and distant recurrence as competing risks. Similarly, the cumulative incidence of distant recurrence was estimated considering death and loco-regional recurrence as competing risks. The Fine-Gray regression model was used to estimate the effect of baseline covariates on the competing risks end-point [40]. Gray's test was used to compare cumulative incidence across groups as defined by biomarker dichotomization (e.g., high vs low). All reported $\mathrm{p}$ values are two-sided and $\mathrm{p}$ values less than $5 \%$ were considered to be significant. Analyses were performed with the R software (version 4.0.2) [41].

\section{Results}

\section{Patient characteristics}

Table 1 summarizes the detailed clinicopathological characteristics and PET imaging parameters of the 303 patients. The median age was 50 years (range, 23-96) and more than one-half of patients had histologically proven regional lymph node metastasis (54\%). All patients underwent breast surgery either after neoadjuvant therapy (51\%) or before adjuvant chemotherapy (38\%) alone or in combination with anti-HER2 treatment $(4 \%)$. The majority of patients $(96 \%)$ received external beam radiation therapy and about two-third received adjuvant endocrine therapy (63\%). On histopathological evaluation, $23 \%(n=70)$ of tumors were classified as luminal $A, 30 \%(n=90)$ as luminal $B, 16 \%(n=50)$ as HER2+, and 31\% $(n=93)$ as triple-negative breast cancer (TNBC). The median follow up was 6.2 years (95\% CI 5.1-7.2 years). Fifty-six (18\%) patients developed tumor recurrence and $35(12 \%)$ died during the follow-up period. The distribution of PET features is described in supplemental Fig. 1.

\section{Association with outcomes}

Disease control. According to predictiveness curves, the cut-off values to predict 5y-RFS were as follows: high tumor SUVmax when $>8.6$, high TMTV when $>20 \mathrm{~cm} 3$, high SLR when $>0.76$, and high BLR when $>0.76$ (supplemental Fig. 2). When applying these cut-off values, 143 (47\%), 66 (22\%), 113 (37\%), and 130 (43\%) patients had high tumor SUVmax, high TMTV, high SLR, and high BLR, respectively (Fig. 2). We investigated the ability of PET biomarkers and classical clinicopathological factors to predict 5y-RFS. The 5-year probability of disease control was $86 \%$ (95\% CI 82-90\%). The results of univariable and multivariable Cox regression models are presented in Table 2. In univariable analysis, advanced tumor stage (T3 and T4), lymph node involvement $(\mathrm{N}+)$, high TMTV, and high SLR were associated with a significantly higher 5-year risk of recurrence (Fig. 3). In multivariable analysis, lymph node involvement (HR 4.2, 95\% CI 1.7-10.3), high TMTV 
(HR 2.4, 95\% CI 1.3-4.5), and high SLR (HR 1.9, 95\% CI 1.0-3.6) remained independent statistically significant prognostic factors for $5 \mathrm{y}$ - RFS. Finally, TMTV and SLR added significant prognostic values to the multivariable model obtained with the backward elimination process for 5y-RFS $(\mathrm{p}=0.03$ and 0.04 , respectively; supplemental Table 1).

Loco-regional and systemic control. The 5-year probability of loco-regional and systemic control was 94\% (95\% CI 91-97\%) and 89\% (95\% CI 85-92\%), respectively. Most distant recurrences occurred in the bones (48\%), extra-regional lymph nodes (41\%), lungs (27\%), liver (25\%), and brain (14\%). The number of metastases at time of distant recurrence was $\leq 3$ in $17 \%$, between 4 and 10 in $20 \%$ and $>10$ in $63 \%$ of patients. Oligo-metastatic disease, defined as $\leq 5$ lesions in $\leq 3$ organs [42], was detected in $29 \%$ of patients with distant recurrence.

In multivariable analysis for 5y-LRFS, lymph node involvement (HR 2.9, 95\% CI 1.2-7.4) and high TMTV (HR 3.0, 95\% CI 1.5-6.2) were independent statistically significant prognostic factors for 5y-LRFS (supplemental Table 2, supplemental Fig. 3). Models adapted to a competing risks environment for the cumulative incidence of loco-regional recurrence (supplemental Table 3) highlighted the prognostic significance of tumor SUVmax, TMTV, and SLR in univariable analysis but not BLR. However, none of these markers remained independent prognostic factors in multivariable analysis. The presence of a high SLR failed to reach the limit of statistical significance despite consequent hazard ratios and indicative confidence intervals with Cox or Fine and Gray models. Furthermore, TMTV provides additional prognostic value to the multivariable model obtained with backward elimination for 5y-LRFS ( $\mathrm{p}<0$. 01, supplemental Table 1) whereas SLR did not $(p=0.07)$. In multivariable analysis for 5y-DRFS, lymph node involvement and high SLR were independent statistically significant prognostic factors for 5y-DRFS (supplement Table 4, supplemental Fig. 4). The same directions of the effects were observed when conducting a competing risks analysis on the distant recurrence endpoint (supplemental Table 5) with TMTV (SHR 2.6, 95\% CI 0.9-4.3) and SLR (SHR 2.8, 95\% CI 1.3-5.0). Lastly, SLR added significant prognostic value to the multivariable model obtained with backward elimination for 5y-DRFS ( $\mathrm{p}<0.01$, supplemental Table 1), unlike TMTV $(\mathrm{p}=0.06)$.

Overall, tumor SUVmax values and BLR, as well as other clinical and pathological parameters, except lymph node involvement, were not considered to be statistically independent prognostic factors for 5y-RFS, 5yLRFS, and 5y-DRFS.

\section{Association between biomarkers}

Relationships between PET biomarkers and continuous clinicopathological variables of interest (age, Ki67, CA 15-3, and CEA), are presented in supplemental Fig. 5. No strong explanatory relationship emerged 
from our analysis. However, we found several moderate but significant correlations between tumor SUVmax and TMTV (rho = 0.35), SLR and BLR (rho =0.38), age and BLR (rho $=-0.42, \mathrm{p}<0.05$ ), and Ki67 and tumor SUVmax $($ rho $=0.37, \mathrm{p}<0.05)($ supplemental Fig. 6).

To evaluate the cross-talk between tumor and its immune microenvironment and lymphoid organs, we determined whether there was any association between spleen or bone marrow metabolism and histopathological findings, such as stromal TILs, intra-tumoral necrosis, histologic grade, and mitotic activity index. SLR was significantly higher $(\mathrm{p}<0.01)$ in patients with low stromal TILS compared to patients with high stromal TILs (supplemental Table 6). This inverse association between SLR and stromal TILs remained significant in the molecular subset analysis, but only in TNBC $(\mathrm{p}<0.01)$ and luminal A $(\mathrm{p}=0.03)$ patients (supplemental Table 7, supplemental Fig. 7). In the logistic regression performed to provide a quantitative value for the strength of this association after adjustment for other variables (to remove confounding effects), high SLR remained the only independent factor for low stromal TILs (odds ratio [OR] 2.8, 95\%CI 1.4-5.7) (Fig. 4). Regarding other pathological features, as expected, tumor SUVmax was significantly associated with histologic grade and showed a strong trend towards significance with mitotic activity index (supplemental Fig. 8f). No other relevant association was detected between necrosis and tumor SUVmax or SLR.

\section{Discussion}

We have shown that total metabolic tumor volume (TMTV) and spleen-to-liver glucose uptake ratio (SLR) on staging [18F]-FDG PET/CT are independent prognostic biomarkers for 5-year recurrence in a retrospective cohort of 303 patients. These two biomarkers appear to reliably predict tumor recurrence as well as lymph node involvement $(\mathrm{N})$.

Our results are consistent with previously published data in various tumor types, suggesting that a new milestone has been reached in this field [20, 23, 26, 31]. In the specific setting of breast cancer, Şahin et al. demonstrated that FDG uptake in the spleen or bone marrow on pretreatment PET imaging was higher in breast cancer patients than in patients without any cancer (control group) [22]. Interestingly, another important result demonstrated by this study was the strong association between spleen glucose metabolism and the triplenegative tumor subtype, which is consistent with our findings. Bang et al. showed that tumor size (T stage), ER status, and spleen glucose metabolism on baseline [18F]-FDG PET/CT imaging were independent prognostic factors for disease-free survival in 153 patients with breast cancer of NST undergoing upfront surgery followed by adjuvant therapy [23]. Our work therefore supports the prognostic significance of spleen metabolism and justifies external validation on an independent cohort of patients with breast cancer of NST.

Demonstration that lymphoid tissue glucose metabolism is an imaging biomarker correlated with RFS in breast cancer is an interesting finding, consistent with data published in the literature. In a recent study, Lee et al. explored the association between RFS or DRFS (systemic control) and PET biomarkers including bone 
marrow metabolism (BLR) in 345 patients with invasive ductal (89\%) or lobular (11\%) breast carcinoma treated by upfront surgery prior to adjuvant therapy [24]. They confirmed that metabolic tumor volume and noninvasive measurement of glucose metabolism on nontumoral lymphoid tissues can both be used to predict recurrence, especially the onset of metastasis. These authors demonstrated that total lesion glycolysis (TLG) and bone marrow-to-liver uptake ratio (BLR) were relevant biomarkers achieving the best performance to predict RFS and DRFS status. However, as acknowledged by the authors, spleen uptake was not studied and its prognostic value can therefore not be compared with bone marrow uptake. In our cohort, we did not find any association between bone marrow metabolism and risk of recurrence. Interestingly, we observed a correlation between bone marrow and splenic metabolism supporting the hypothesis of a pathophysiologic mechanism and a common systemic inflammatory state. Consequently, we suggest that the predictive value of spleen glucose metabolism may outperform that of bone marrow glucose metabolism.

Our results support the existence of cross-talk between tumor and its microenvironment and lymphoid tissues. Our observations suggest that spleen glucose metabolism is higher in patients who experienced recurrence compared to patients without recurrence, but also higher in patients with low stromal TILs compared to patients with high stromal TILs. The clinical relevance of TILs in breast cancer has been clearly established, since lymphocyte-predominant breast cancer is associated with favorable clinical outcome, particularly in TNBC patients [43]. Furthermore, breast cancer progression is closely related to the tumor microenvironment (TME), including tumor, immune, stromal, and bone marrow and/or spleen-derived inflammatory cells [44]. In addition to TILs, MDSCs are also major cell components in the TME, the functioning of which blocks both lymphocyte infiltration and $\mathrm{T}$ cell function by inducing the recruitment of regulatory $\mathrm{T}$ cells (Tregs). In patients with poor prognosis, tumor conditions alter myelopoiesis, leading to MDSC expansion from lymphoid organs (bone marrow and spleen), which then infiltrate the TME. There therefore appears to be a balance within the complex TME between TILs, involved in the antitumor immune response, and tumor-infiltrating myeloid cells that block tumor immunity [45]. Theoretically, high spleen glucose metabolism might be related to an immunosuppressive TME with a lack of TILs and an abundance of suppressive immune cells such as MDSCs, especially for TNBC. However, mechanisms causing the high spleen glucose metabolism could be multiple, with, for example, cancer-related inflammation, which requires further analyses to explore the relationship between SLR and established markers of cancer-related inflammatory, such as C-reactive protein, leukocytes, and neutrophils in the peripheral blood [46].

The strengths of our study are the large sample size and the long follow-up. The main limitation concerns the retrospective nature and single-center study. In order to study a representative real-life patient population, we did not exclude patients with small tumors (T1, less than $20 \mathrm{~mm}: \mathrm{n}=86,28 \%$ ) and our findings are consequently subject to a partial volume effect bias. Small tumors with low metabolic activity are usually difficult to delineate due to the surrounding physiologic uptake. We also used an isocontour method with a threshold of $41 \%$ as proposed in the EANMguidelines [36], but this approach could result in inaccurate tumor 
segmentation in small tumors [47]. Still, tumor segmentation results of such cases were visually acceptable, highly reproducible between observers and therefore did not affect the metabolic assessment of non-tumoral lymphoid tissues. Moreover, this relatively high number of small tumors is due to the fact that disease stage using [18F]- FDG PET/CT was systematically considered for patients with T1 breast cancer associated with at least one indicative of inherent cancer aggressiveness (clinically positive axillary lymph nodes, aggressive phenotypes as well as TNBC or HER2, clinical symptoms or laboratory values suggesting the presence of metastases), as mentioned in the recent ESMO guidelines [48]. In addition, cut-off values determined by predictiveness curves for each imaging biomarker may have led to an over-estimation of the prognostic performance which should be further validated in an independent external cohort. Finally, as patients were included in the database over a 5-year period, it is possible that progress in surgical or radiation therapy techniques and medical oncology may have resulted in improved patient outcomes.

This study opens up new prospects for the future. Firstly, tumor and lymphoid tissues assessed on baseline [18F]-FDG PET/CT could help to identify patients at higher risk of recurrence, who would require intensified initial therapy and/or close monitoring during follow-up. Secondly, we suggest the potential value of spleen glucose metabolism to predict response to therapies targeting multiple immune compartments within the TME [45]. As stromal TILs are positively associated with PD-L1 expression in TNBC patients [49], and as both of these parameters are correlated with pathological complete response, spleen metabolism could be a marker to identify a subset of patients who may benefit from immune checkpoint inhibitors [50].

The ability of these imaging biomarkers to predict recurrence in addition to gene expression-based ormolecular-based assays needs to be determined. In terms of the cross-talk between the tumor and its TME and lymphoid tissues, our data provide a deeper understanding of how TNBC patients with high spleen glucose metabolism display low stromal TILs, suggesting insufficient immune responses. Future translational studies should focus on the elucidation of the mechanisms involved. Further research is required to identify cancerrelated inflammation and immunosuppressive phenotypes associated with treatment resistance and unfavorable outcomes by the use of quantitative transcriptome analyses of tumors [18], lymphoid tissue biopsies, and immuno-PET imaging [51].

\section{Conclusions}

In this large cohort of patients with non-metastatic breast cancer of NST, we demonstrated that total metabolic tumor volume and spleen metabolism on staging [18F]-FDG PET/CT scans are strong predictors of tumor recurrence. Furthermore, both of these markers may be useful for the stratification of high-risk subpopulations of patients with non-metastatic breast cancer of NST in terms of recurrence. High spleen glucose metabolism was associated with low stromal TILs in the TME, which could block antitumor immunity. 
Further prospective studies using quantitative transcriptome analyses are warranted to investigate this cross-talk between the tumor and its TME and lymphoid tissue metabolism, especially in TNBC.

\section{Supplementary Information}

The online version contains supplementary material available at https://doi.org/10.1007/s00259-021-05322-2.

\section{Author contribution}

All authors made substantial contributions to the design of the work or the acquisition, analysis, or interpretation of data; revised it critically for important intellectual content; approved the version to be published; and agreed to be accountable for all aspects of the work in ensuring that questions related to the accuracy or integrity of any part of the work are appropriately investigated and resolved. Material preparation, data collection, and analysis were performed by R-D. Seban, A. Latouche, N. Deleval, F-C. Bidard, and L. Champion. The first draft of the manuscript was written by R-D Seban, F-C. Bidard, and L. Champion. All authors commented on previous versions of the manuscript. All authors read and approved the manuscript.

\section{Declarations}

Ethics approval All procedures performed in this study were in accordance with the ethical standards of the institutional research committee and with the 1964 Helsinki declaration. Consent to participate For this type of study (retrospective), formal consent is not required.

Competing interests: The authors declare no competing interests.

\section{References}

1. Desmedt C, Piette F, Loi S, Wang Y, Lallemand F, Haibe-Kains B, et al. Strong time dependence of the 76gene prognostic signature for node-negative breast cancer patients in the TRANSBIG multicenter independent validation series. Clin Cancer Res. 2007;13: 3207-14.

2. Albain KS, Barlow WE, Shak S, Hortobagyi GN, Livingston RB, Yeh I-T, et al. Prognostic and predictive value of the 21-gene recurrence score assay in postmenopausal women with node-positive, oestrogen-receptorpositive breast cancer on chemotherapy: a retrospective analysis of a randomised trial. Lancet Oncol. 2010;11: 55-65. 
3. Lee SH, Ha S, An HJ, Lee JS, Han W, Im S-A, et al. Association between partial-volume corrected SUVmax and Oncotype DX recurrence score in early-stage, ER-positive/HER2-negative invasive breast cancer. Eur J Nucl Med Mol Imaging. 2016;43:1574-84.

4. Buus R, Sestak I, Kronenwett R, Ferree S, Schnabel CA, Baehner FL, et al. Molecular drivers of Oncotype DX, Prosigna, EndoPredict, and the Breast Cancer Index: a TransATAC study. J Clin Oncol. 2021;39:126-35.

5. Ko H, Baghdadi Y, Love C, Sparano JA. Clinical utility of 18FFDG PET/CT in staging localized breast cancer before initiating preoperative systemic therapy. J Natl Compr Cancer Netw. 2020;18:1240-6.

6. Hyland CJ, Varghese F, Yau C, Beckwith H, Khoury K, Varnado W, et al. Use of 18F-FDG PET/CT as an initial staging procedure for stage II-III breast cancer: a multicenter value analysis. J Natl Compr Cancer Netw. 2020;18:1510-7.

7. Nakajo M, Kajiya Y, Kaneko T, Kaneko Y, Takasaki T, Tani A, et al. FDG PET/CT and diffusion-weighted imaging for breast cancer: prognostic value of maximum standardized uptake values and apparent diffusion coefficient values of the primary lesion. Eur J Nucl Med Mol Imaging. 2010;37:2011-20.

8. Groheux D, Giacchetti S, Moretti J-L, Porcher R, Espié M, Lehmann-Che J, et al. Correlation of high 18FFDG uptake to clinical, pathological and biological prognostic factors in breast cancer. Eur J Nucl Med Mol Imaging. 2011;38:426-35.

9. Koo HR, Park JS, Kang KW, Han W, Park IA, Moon WK. Correlation between (18)F-FDG uptake on PET/CT and prognostic factors in triple-negative breast cancer. Eur Radiol. 2015;25:3314-21.

10. Nishimukai A, Inoue N, Kira A, Takeda M, Morimoto K, Araki K, et al. Tumor size and proliferative marker geminin rather than Ki67 expression levels significantly associated with maximum uptake of 18Fdeoxyglucose levels on positron emission tomography for breast cancers. PLoS One. 2017;12:e184508.

11. Kitajima K, Yamano T, Fukushima K, Miyoshi Y, Hirota S, Kawanaka Y, et al. Correlation of the SUVmax of FDG-PET and ADC values of diffusion-weighted MR imaging with pathologic prognostic factors in breast carcinoma. Eur J Radiol. 2016;85: 943-9.

12. Yoo J, Yoon H-J, Kim BS. Prognostic value of primary tumor SUVmax on F-18 FDG PET/CT compared with semi-quantitative tumor uptake on Tc-99m sestamibi breast-specific gamma imaging in invasive ductal breast cancer. Ann Nucl Med. 2017;31:19-28.

13. DiaoW, Tian F, Jia Z. The prognostic value of SUVmaxmeasuring on primary lesion and ALN by $18 \mathrm{~F}-\mathrm{FDG}$ PET or PET/CT in patients with breast cancer. Eur J Radiol. 2018;105:1-7.

14. Jiménez-Ballvé A, García García-Esquinas M, Salsidua-Arroyo O, Serrano-Palacio A, García-Sáenz JA, Ortega Candil A, et al. Prognostic value of metabolic tumour volume and total lesion glycolysis in 18F-FDG PET/CT scans in locally advanced breast cancer staging. Rev Esp Med Nucl Imagen Mol. 2016;35:365-72. 
15. Higuchi T, Fujimoto Y, Ozawa H, Bun A, Fukui R, Miyagawa Y, et al. Significance of metabolic tumor volume at baseline and reduction of mean standardized uptake value in 18F-FDG-PET/CT imaging for predicting pathological complete response in breast cancers treated with preoperative chemotherapy. Ann Surg Oncol. 2019;26:2175-83.

16. Lemarignier C, Martineau A, Teixeira L, Vercellino L, Espié M, Merlet P, et al. Correlation between tumour characteristics, SUV measurements, metabolic tumour volume, TLG and textural features assessed with 18F-FDG PET in a large cohort of oestrogen receptor-positive breast cancer patients. Eur J Nucl Med Mol Imaging. 2017;44:1145-54.

17. Seban R-D, Robert C, Dercle L, Yeh R, Dunant A, Reuze S, et al. Increased bone marrow SUVmax on 18FFDG PET is associated with higher pelvic treatment failure in patients with cervical cancer treated by chemoradiotherapy and brachytherapy. Oncoimmunology. 2019;8:e1574197.

18. Seban R-D, Nemer JS,Marabelle A, Yeh R, Deutsch E, Ammari S, et al. Prognostic and theranostic 18FFDG PET biomarkers for anti- PD1 immunotherapy in metastatic melanoma: association with outcome and transcriptomics. Eur J Nucl Med Mol Imaging. 2019;46: 2298-310.

19. Seban R-D, Moya-Plana A, Antonios L, Yeh R, Marabelle A, Deutsch E, et al. Prognostic 18F-FDG PET biomarkers in metastatic mucosal and cutaneous melanoma treated with immune checkpoint inhibitors targeting PD-1 and CTLA-4. Eur J Nucl Med Mol Imaging. 2020.

20. Wong A, Callahan J, Keyaerts M, Neyns B, Mangana J, Aberle S, et al. 18F-FDG PET/CT based spleen to liver ratio associates with clinical outcome to ipilimumab in patients with metastatic melanoma. Cancer Imaging. 2020;20:36.

21. Prigent K, Lasnon C, Ezine E, Janson M, Coudrais N, Joly E, et al. Assessing immune organs on 18F-FDG PET/CT imaging for therapy monitoring of immune checkpoint inhibitors: inter-observer variability, prognostic value and evolution during the treatment course of melanoma patients. Eur J Nucl Med Mol Imaging. 2021.

22. Şahin E, Elboğa U. Relationship between reticuloendothelial systems' FDG uptake level and clinicopathological features in patient with invasive ductal breast cancer. Radiol Med. 2017;122:785-92.

23. Bang J-I, Yoon H-J, Kim BS. Clinical utility of FDG uptake within reticuloendothelial system on F-18 FDG PET/CT for prediction of tumor recurrence in breast cancer. PLoS One. 2018;13:e0208861.

24. Lee JW, Kim SY, Han SW, Lee JE, Lee HJ, Heo NH, et al. [18F]FDG uptake of bone marrow on PET/CT for predicting distant recurrence in breast cancer patients after surgical resection. EJNMMI Res. 2020;10:72.

25. Lee JW, Lee M-S, Chung IK, Son MW, Cho YS, Lee SM. Clinical implication of FDG uptake of bone marrow on PET/CT in gastric cancer patients with surgical resection. World J Gastroenterol. 2017;23:2385-95. 
26. Yoon H-J, Kim BS, Moon CM, Yoo J, Lee KE, Kim Y. Prognostic value of diffuse splenic FDG uptake on PET/CT in patients with gastric cancer. PLoS One. 2018;13:e0196110.

27. Lee JW, Choi JS, Lyu J, Lee SM. Prognostic significance of 18Ffluorodeoxyglucose uptake of bone marrow measured on positron emission tomography in patients with small cell lung cancer. Lung Cancer. 2018;118:41-7.

28. Jeong H, Hwang I, Kang SH, Shin HC, Kwon SY. Tumorassociated macrophages as potential prognostic biomarkers of invasive breast cancer. J Breast Cancer. 2019;22:38-51.

29. Hashemi V, Maleki LA, Esmaily M, Masjedi A, Ghalamfarsa G, Namdar A, et al. Regulatory T cells in breast cancer as a potent anticancer therapeutic target. Int Immunopharmacol. 2020;78:106087.

30. Alshetaiwi H, Pervolarakis N, McIntyre LL, Ma D, Nguyen Q, Rath JA, et al. Defining the emergence ofmyeloid-derived suppressor cells in breast cancer using single-cell transcriptomics. Sci Immunol. 2020;5.

31. Seban R-D, Champion L, Schwartz LH, Dercle L. Spleen glucose metabolism on [18F]-FDG PET/CT: a dynamic double-edged biomarker predicting outcome in cancer patients. Eur J Nucl Med Mol Imaging. 2021.

32. Amin MB, Greene FL, Edge SB, Compton CC, Gershenwald JE, Brookland RK, et al. The eighth edition AJCC cancer staging manual: continuing to build a bridge from a population-based to a more "personalized" approach to cancer staging. CA Cancer J Clin. 2017;67:93-9.

33. Cserni G, Chmielik E, Cserni B, Tot T. The new TNM-based staging of breast cancer. Virchows Arch. 2018;472:697-703.

34. Loi S, Sirtaine N, Piette F, Salgado R, Viale G, Van Eenoo F, et al. Prognostic and predictive value of tumor-infiltrating lymphocytes in a phase III randomized adjuvant breast cancer trial in nodepositive breast cancer comparing the addition of docetaxel to doxorubicin with doxorubicin-based chemotherapy: BIG 02-98. J Clin Oncol. 2013;31:860-7.

35. Goldhirsch A, Wood WC, Coates AS, Gelber RD, Thürlimann B, Senn H-J, et al. Strategies for subtypesdealing with the diversity of breast cancer: highlights of the St. Gallen international expert consensus on the primary therapy of early breast cancer 2011. Ann Oncol. 2011;22:1736-47.

36. Boellaard R, Delgado-Bolton R, Oyen WJG, Giammarile F, Tatsch K, Eschner W, et al. FDG PET/CT: EANM procedure guidelines for tumour imaging: version 2.0. Eur J Nucl Med Mol Imaging. 2015;42:328-54. 37. Schemper M, Smith TL. A note on quantifying follow-up in studies of failure time. Control Clin Trials. 1996;17:343-6.

38. Viallon V, Latouche A. Discrimination measures for survival outcomes: connection between the AUC and the predictiveness curve. Biom J. 2011;53:217-36. 
39. Empereur-Mot C, Guillemain H, Latouche A, Zagury J-F, Viallon V, Montes M. Predictiveness curves in virtual screening. J Cheminform. 2015;7:52.

40. Latouche A, Allignol A, Beyersmann J, Labopin M, Fine JP. A competing risks analysis should report results on all cause-specific hazards and cumulative incidence functions. J Clin Epidemiol. 2013;66:648-53.

41. R Core Team. R: A Language and Environment for Statistical Computing [Internet]. Vienna, Austria: 2020; Available from: https://www.R-project.org

42. Milano MT, Katz AW, Zhang H, Okunieff P. Oligometastases treated with stereotactic body radiotherapy: long-term follow-up of prospective study. Int J Radiat Oncol Biol Phys. 2012;83:878- 86.

43. Denkert C, von Minckwitz G, Darb-Esfahani S, Lederer B, Heppner BI, Weber KE, et al. Tumourinfiltrating lymphocytes and prognosis in different subtypes of breast cancer: a pooled analysis of 3771 patients treated with neoadjuvant therapy. Lancet Oncol. 2018;19:40-50.

44. Annaratone L, Cascardi E, Vissio E, Sarotto I, Chmielik E, Sapino A, et al. The multifaceted nature of tumor microenvironment in breast carcinomas. Pathobiology. 2020;87:125-42.

45. Christmas BJ, Rafie CI, Hopkins AC, Scott BA, Ma HS, Cruz KA, et al. Entinostat converts immuneresistant breast and pancreatic cancers into checkpoint-responsive tumors by reprogramming tumor-infiltrating MDSCs. Cancer Immunol Res. 2018;6:1561-77.

46. Seban R-D, Assié J-B, Giroux-Leprieur E, Massiani M-A, Soussan M, Bonardel G, et al. Association of the metabolic score using baseline FDG-PET/CT and dNLR with immunotherapy outcomes in advanced NSCLC patients treated with first-line pembrolizumab. Cancers. Multidisciplinary Digital Publishing Institute; 2020;12: 2234.

47. Tylski P, Stute S, Grotus N, Doyeux K, Hapdey S, Gardin I, et al. Comparative assessment of methods for estimating tumor volume and standardized uptake value in (18)F-FDG PET. J Nucl Med. 2010;51:268-76.

48. Cardoso F, Kyriakides S, Ohno S, Penault-Llorca F, Poortmans P, Rubio IT, et al. Early breast cancer: ESMO clinical practice guidelines for diagnosis, treatment and follow-up $\dagger$. Annals of Oncology Elsevier. 2019;30:1194-220.

49. McLemore LE, Janakiram M, Albanese J, Shapiro N, Lo Y, Zang X, et al. An immunoscore using PD-L1, CD68, and tumorinfiltrating lymphocytes (TILs) to predict response to neoadjuvant chemotherapy in invasive breast cancer. Appl Immunohistochem Mol Morphol. 2018;26:611-9.

50. Wimberly H, Brown JR, Schalper K, Haack H, Silver MR, Nixon C, et al. PD-L1 expression correlates with tumor-infiltrating lymphocytes and response to neoadjuvant chemotherapy in breast cancer. Cancer Immunol Res. 2015;3:326-32. 
51. Bensch F, van der Veen EL, Lub-de Hooge MN, Jorritsma-Smit A, Boellaard R, Kok IC, et al. 89Zratezolizumab imaging as a noninvasive approach to assess clinical response to PD-L1 blockade in cancer. Nat Med. 2018;24:1852-8. 


\begin{tabular}{ccccc}
$\begin{array}{c}\text { All patients } \\
\mathbf{n = 3 0 3} \text { pts }\end{array}$ & $\begin{array}{c}\text { Luminal A } \\
\text { n=70 pts }\end{array}$ & $\begin{array}{c}\text { Luminal B } \\
\text { n=90 pts }\end{array}$ & $\begin{array}{c}\text { HER2 } \\
\text { n=50 pts }\end{array}$ & $\begin{array}{c}\text { TNBC } \\
\text { n=93 pts }\end{array}$ \\
\hline
\end{tabular}

Median [range], n (\%)

\begin{tabular}{|c|c|c|c|c|c|}
\hline \multicolumn{6}{|l|}{$\begin{array}{l}\text { CLINICOPATHOLOGICAL } \\
\text { CHARACTERISTICS }\end{array}$} \\
\hline \multicolumn{6}{|l|}{ Demographic parameters } \\
\hline Age (years) & $50[23-96]$ & $53[35-91]$ & $50[23-96]$ & $46[25-82]$ & $49[24-86]$ \\
\hline \multicolumn{6}{|l|}{ Post-menopausal status } \\
\hline \multicolumn{6}{|l|}{ TNM Stage (AJCC classification) } \\
\hline $\mathrm{T} 1$ & $86(28)$ & $25(36)$ & $24(27)$ & $14(28)$ & $25(27)$ \\
\hline $\mathrm{T} 2$ & $144(48)$ & $33(47)$ & $47(52)$ & $22(44)$ & $43(46)$ \\
\hline $\mathrm{T} 3$ & $54(18)$ & $10(14)$ & $17(19)$ & $10(20)$ & $17(18)$ \\
\hline $\mathrm{T} 4$ & $11(4)$ & $2(3)$ & $2(2)$ & $2(4)$ & $5(5)$ \\
\hline $\mathrm{N}+$ & $164(54)$ & $40(57)$ & $54(60)$ & $27(54)$ & $43(46)$ \\
\hline \multicolumn{6}{|l|}{ Histologic parameters } \\
\hline Vascular invasion & $42(14)$ & $12(17)$ & $11(12)$ & $4(8)$ & $15(16)$ \\
\hline Necrosis & $75(25)$ & $10(14)$ & $16(18)$ & $12(24)$ & $37(40)$ \\
\hline Low stromal TILs $(<50 \%)$ & $213(70)$ & $61(87)$ & $68(76)$ & $36(72)$ & $58(62)$ \\
\hline \multicolumn{6}{|l|}{ Histologic grade } \\
\hline I-II & $136(45)$ & $66(94)$ & $35(39)$ & $23(46)$ & $12(13)$ \\
\hline III & $167(55)$ & $4(6)$ & $55(61)$ & $27(54)$ & $81(87)$ \\
\hline Ki67 & $38[1-95]$ & $15[1-20]$ & 35 [20-95] & $35[5-85]$ & $60[20-90]$ \\
\hline High mitotic index ( $\geq 13$ mitosis $/ 2 \mathrm{~mm}^{2}$ ) & $123(41)$ & $0(0)$ & $34(38)$ & $19(38)$ & $63(68)$ \\
\hline \multicolumn{6}{|l|}{ Tumor markers $(\mathrm{ng} / \mathrm{mL})$} \\
\hline Cancer Antigen 15-3 (CA 15-3) $>30 \mathrm{U} / \mathrm{mL}$ & $60(20)$ & $13(19)$ & $19(21)$ & $10(20)$ & $18(19)$ \\
\hline CarcinoEmbryonic Antigen $(\mathrm{CEA})>5 \mu \mathrm{g} / \mathrm{L}$ & $20(7)$ & $8(11)$ & $4(4)$ & $6(12)$ & $2(2)$ \\
\hline \multicolumn{6}{|l|}{ PET IMAGING CHARACTERISTICS } \\
\hline \multicolumn{6}{|l|}{ Tumor glucose uptake } \\
\hline Tumor SUVmax & $8.1[1.0-43.0]$ & $5.6[1.4-20.9]$ & $7.9[1.0-43.0]$ & $7.8[2.7-18.4]$ & $10.4[1.7-24.6]$ \\
\hline \multicolumn{6}{|l|}{ Metabolic tumor burden $\left(\mathrm{cm}^{3}\right)$} \\
\hline Total Metabolic Tumor Volume (TMTV) & $6.2[0.4-351.0]$ & $4.2[0.4-51.7]$ & $8.0[0.4-351.0]$ & $5.2[1.0-116.5]$ & $6.2[0.7-308.5]$ \\
\hline \multicolumn{6}{|l|}{ Lymphoid tissue metabolism } \\
\hline Spleen to liver SUVmax ratio (SLR) & $0.75[0.51-1.07]$ & $0.75[0.51-1.07]$ & $0.75[0.59-1.02]$ & $0.73[0.52-0.91]$ & $0.75[0.57-0.99]$ \\
\hline Bone marrow to liver SUVmax ratio (BLR) & $0.76[0.39-1.18]$ & $0.78[0.39-1.15]$ & $0.75[0.55-1.06]$ & $0.76[0.44-1.03]$ & $0.76[0.51-1.18]$ \\
\hline \multicolumn{6}{|l|}{ TREATMENT } \\
\hline Surgery & $303(100)$ & $70(100)$ & $90(100)$ & $50(100)$ & $93(100)$ \\
\hline \multicolumn{6}{|l|}{ Neo-adjuvant therapy } \\
\hline Chemotherapy & $151(50)$ & $17(24)$ & $48(53)$ & $29(58)$ & $57(61)$ \\
\hline Anti-HER2 & $31(10)$ & $0(0)$ & $0(0)$ & $29(58)$ & $0(0)$ \\
\hline Endocrine therapy & $5(2)$ & $3(4)$ & $2(2)$ & $0(0)$ & $0(0)$ \\
\hline \multicolumn{6}{|l|}{ Adjuvant therapy } \\
\hline Chemotherapy & $116(38)$ & $31(44)$ & $35(39)$ & $20(40)$ & $37(40)$ \\
\hline Anti-HER2 & $13(4)$ & $0(0)$ & $0(0)$ & $20(40)$ & $0(0)$ \\
\hline Endocrine therapy & $192(63)$ & $67(96)$ & $90(100)$ & $31(62)$ & $0(0)$ \\
\hline Radiotherapy & $292(96)$ & $63(90$ & $89(99)$ & $50(100)$ & $90(97)$ \\
\hline
\end{tabular}


Table 2: Prognostic significance of biomarkers for 5-year recurrence-free survival in univariable and multivariable analyses.

\begin{tabular}{|c|c|c|c|c|}
\hline \multirow{3}{*}{$\begin{array}{l}\mathrm{n}=303 \mathrm{pts} \\
\text { Events }=44\end{array}$} & \multicolumn{4}{|c|}{ 5-YEAR RECURRENCE-FREE SURVIVAL } \\
\hline & \multicolumn{2}{|c|}{ Univariable } & \multicolumn{2}{|c|}{ Multivariable } \\
\hline & HR $(95 \%$ CI $)$ & p value & HR $(95 \%$ CI) & p value \\
\hline Age $<40$ years $(v s \geq 40)$ & $0.9(0.4-1.9)$ & 0.72 & - & - \\
\hline Post-menopausal (vs pre-menopausal) & $1.3(0.7-2.5)$ & 0.34 & - & - \\
\hline T stage III-IV (vs I-II) & $2.9(1.6-5.3)$ & $<0.01$ & - & - \\
\hline $\mathbf{N}+(\mathbf{v s} \mathbf{N}-)$ & $5.9(2.5-14.1)$ & $<0.01$ & $4.2(1.7-10.3)$ & $<0.01$ \\
\hline Molecular sub-types & & & - & - \\
\hline Luminal A & 1.0 (Reference) & - & - & - \\
\hline Luminal B & $1.7(0.7-2.2)$ & 0.57 & - & - \\
\hline HER2 & $1.1(0.4-3.0)$ & 0.79 & - & - \\
\hline TNBC & $2.0(0.8-5.0)$ & 0.13 & - & - \\
\hline Vascular invasion (yes vs no) & $1.2(0.5-2.7)$ & 0.63 & - & - \\
\hline Histologic grade 3 (vs 1-2) & $2.1(0.8-5.5)$ & 0.13 & - & - \\
\hline Low stromal TILs (vs high) & $1.4(0.7-2.7)$ & 0.17 & - & - \\
\hline Tumor SUVmax > $8.6(\leq 8.6)$ & $2.2(0.8-4.8)$ & 0.10 & - & - \\
\hline TMTV $>20 \mathrm{~cm}^{3}\left(\mathrm{vs} \leq 20 \mathrm{~cm}^{3}\right)$ & $4.8(2.6-8.8)$ & $<0.01$ & $2.4(1.3-4.5)$ & $<0.01$ \\
\hline SLR $>0.76(\leq 0.76)$ & $2.8(1.5-5.2)$ & $<0.01$ & $1.9(1.0-3.6)$ & 0.04 \\
\hline BLR $>0.78(\leq 0.78)$ & $1.3(0.7-2.4)$ & 0.32 & - & - \\
\hline
\end{tabular}

Abbreviations: hazard ratio (HR), confidence interval (CI), tumor (T), node involvement $(N)$, tumor-infiltrating lymphocytes (TILs), maximum standardized uptake value (SUVmax), total metabolic tumor volume (TMTV), spleen-toliver ratio (SLR), bone marrow-to-liver ratio (BLR). 
Figure 1: Flow chart.

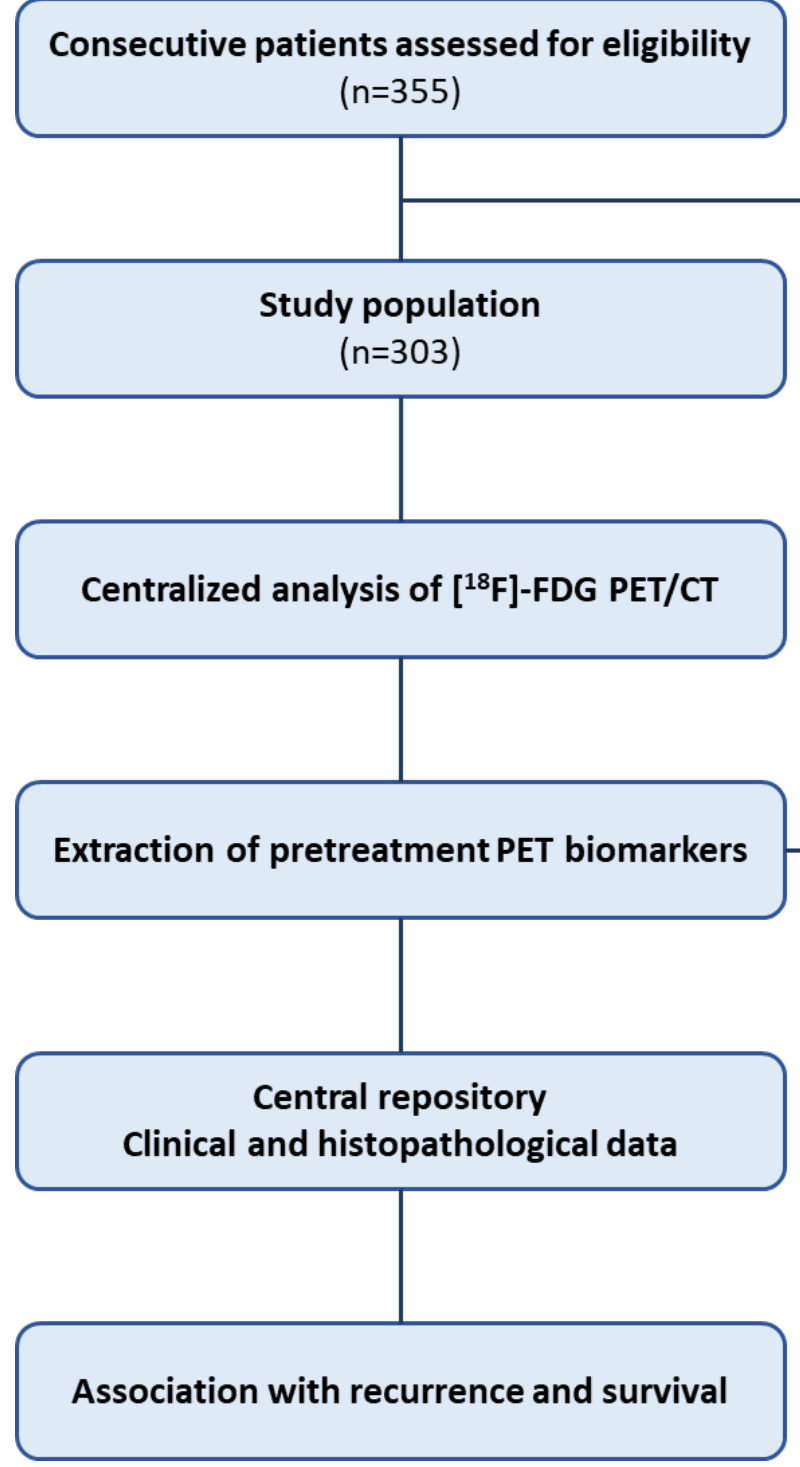

\section{Patients who met exclusion criteria}

Metastatic disease

Lost to follow-up

No mesurable disease / no FDG-avid tumor

Other primary malignancies

Treatment or disease influencing lymphoid tissues

Opposition to participate in medical research $(n=52)$

$(n=13)$

$(n=9)$

$(n=8)$

$(n=7)$

$(n=5)$

$(n=10)$

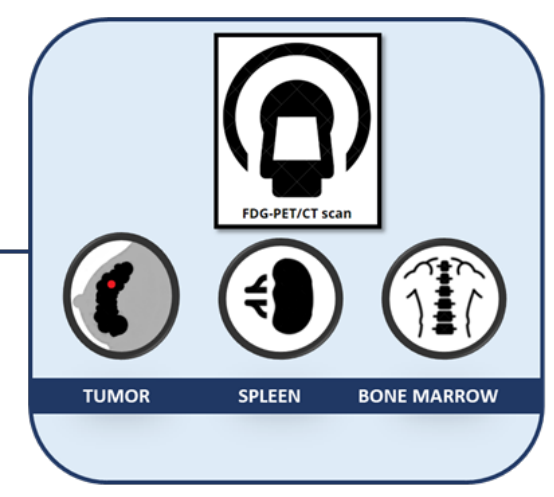


Figure 2: Summary of key results of each analysis (association between TMTV or SLR on $\left[{ }^{18}\right.$ F]-FDG PET and prognosis or stromal TILs on tumor biopsy samples).

\section{TOTAL METABOLIC TUMOR VOLUME (TMTV) and SPLEEN-TO-LIVER UPTAKE RATIO (SLR)}
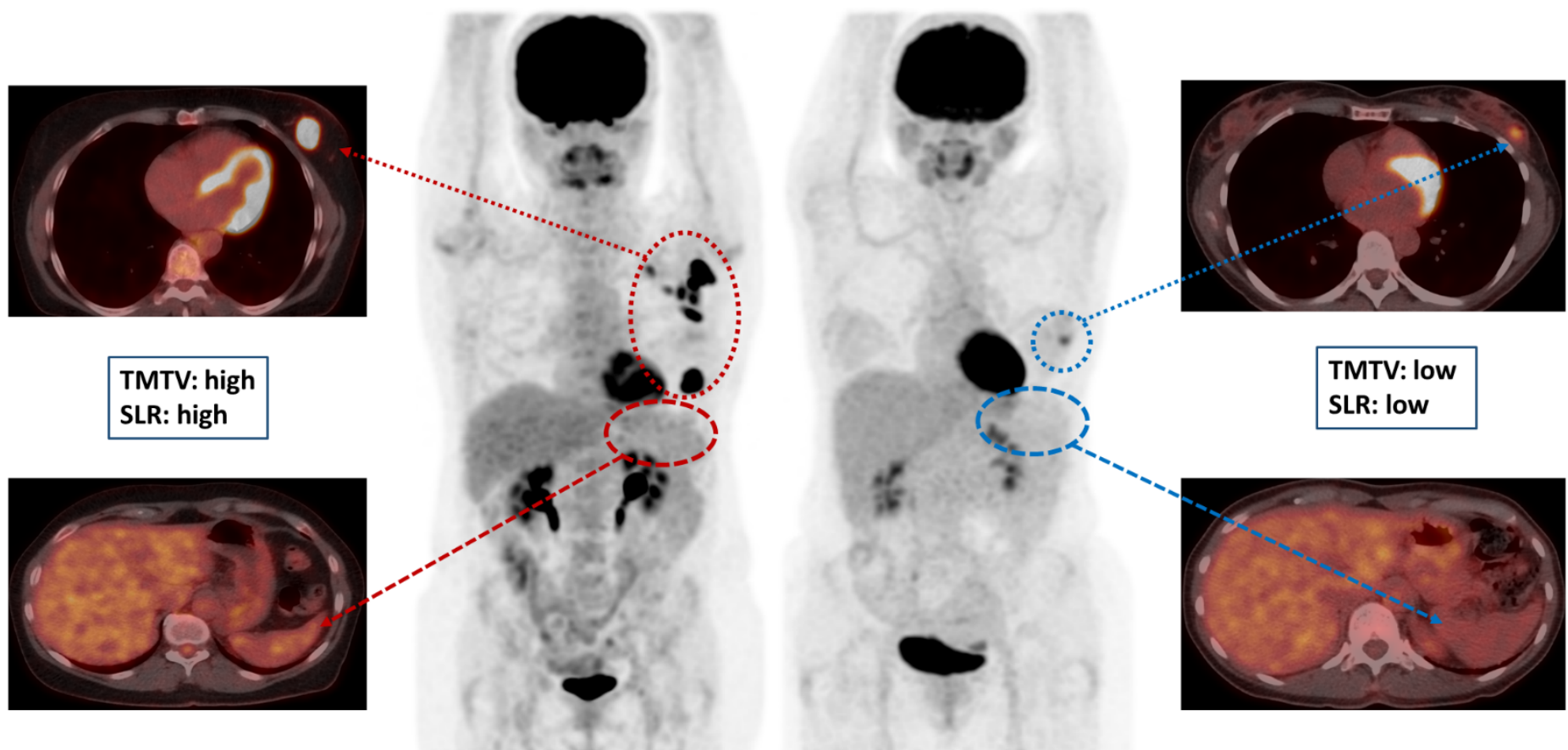
Figure 3: KM curves of RFS according to the Total Metabolic Tumor Volume (high TMTV versus low TMTV) (A) and Spleen-to-Liver Ratio (high SLR versus low SLR) (B).
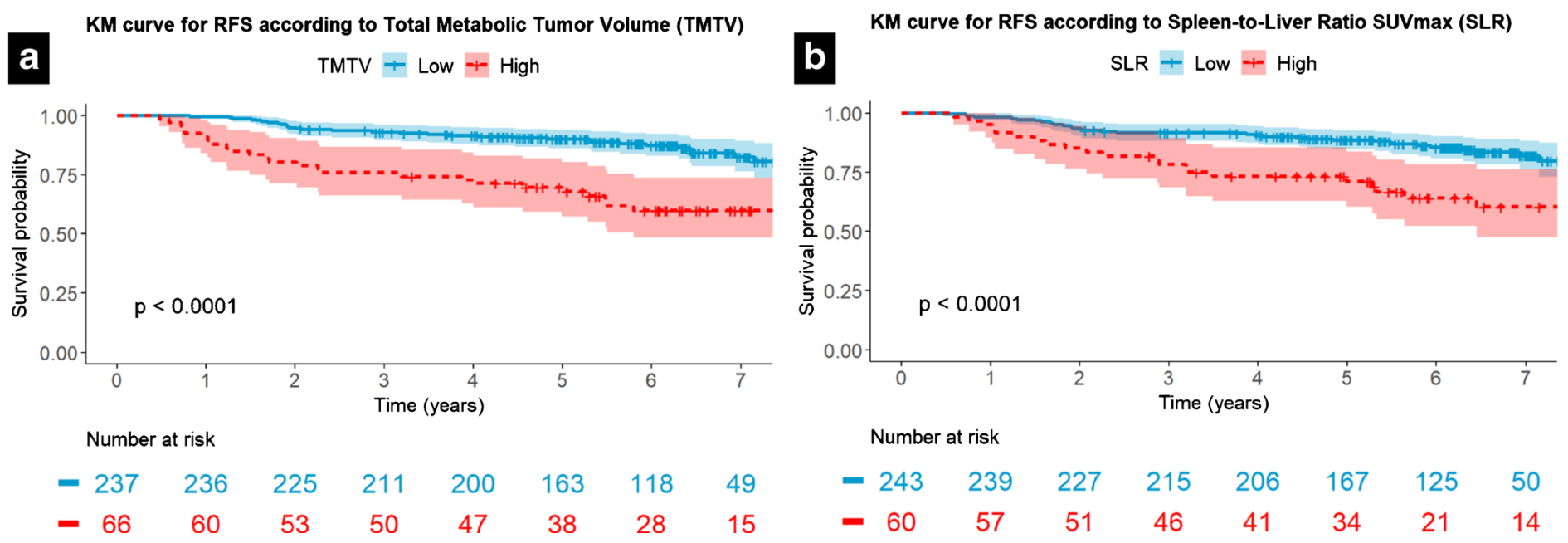
Figure 4: Logistic regression analysis of biomarkers for Low stromal TILs.

\begin{tabular}{|c|c|c|c|c|}
\hline Variable & & Odds ratio & & $\mathrm{p}$ \\
\hline Age $<40$ years $(v s>$ or $=40$ ) & & -1 & $0.89(0.42,1.90)$ & 0.765 \\
\hline Post-menopausal (vs pre-menopausal) & & & $1.07(0.57,2.00)$ & 0.833 \\
\hline T stage (III-IV vs I-II) & & - & $0.82(0.41,1.64)$ & 0.581 \\
\hline Lymph node involvement ( $\mathrm{N}+$ vs $\mathrm{N}-$ ) & & 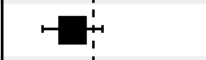 & $0.70(0.42,1.17)$ & 0.172 \\
\hline \multirow[t]{4}{*}{ Molecular subtype } & Luminal A & & Reference & \\
\hline & Luminal B & $\mapsto$ & $0.98(0.44,2.18)$ & 0.961 \\
\hline & HER-2 & 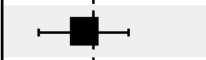 & $0.86(0.40,1.81)$ & 0.687 \\
\hline & Triple negative & 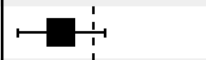 & $0.58(0.27,1.19)$ & 0.142 \\
\hline Vascular invasion (yes vs no) & & & $1.64(0.78,3.61)$ & 0.204 \\
\hline Histologic grade (3 vs 1-2) & & & $0.99(0.60,1.62)$ & 0.958 \\
\hline High Tumor SUVmax (vs low) & & - & $0.71(0.43,1.17)$ & 0.181 \\
\hline High TMTV (vs low) & & in & $1.69(0.85,3.46)$ & 0.138 \\
\hline High SLR (vs low) & & - & $2.76(1.40,5.74)$ & 0.004 \\
\hline High BLR (vs low) & & +1 & $1.06(0.63,1.78)$ & 0.839 \\
\hline
\end{tabular}

